\section{MS2-O5 Phase retrieval for randomly terminated finite crystals}

Joe P.J. Chen ${ }^{1}$, Rick A. Kirian ${ }^{1}$, Richard J. Bean ${ }^{2}$, Kenneth R. Beyerlein ${ }^{2}$, Miriam Barthelmess ${ }^{2}$, Chun Hong Yoon ${ }^{2}$, Fenglin Wang $^{2}$, Flavio Capotondi ${ }^{3}$, Emanuele Pedersoli ${ }^{3}$, Anton Barty ${ }^{2}$, Henry N. Chapman ${ }^{2}$, Philip J. Bones ${ }^{4}$, Romain D. Arnal ${ }^{4}$, Rick P. Millane $^{4}$, John C.H. Spence ${ }^{1}$

1. Department of Physics, Arizona State University, Tempe, Arizona, USA

2. Center for Free-Electron Laser Science, DESY, Hamburg, Germany

3. Fermi, Elettra Sincrotrone Trieste, Trieste, Italy

4. Department of Electrical and Computer Engineering, University of Canterbury, Christchurch, New Zealand

email: jpchen1@mainex1.asu.edu

In serial femtosecond nanocrystallography with x-ray free-electron lasers, diffraction data can be obtained from crystals that have a small number of unit cells [1]. The fewer number of unit cells means that the diffraction patterns from these nanocrystals contain measurable information between the Bragg reflections courtesy of a lattice/shape transform that is no longer delta-like.

By separating the effect of the shape transform from the diffraction via its inherent periodicity about the reciprocal lattice [2], the averaged diffracted intensity from all crystals can be directly converted into the diffracted intensity of a single unit cell. The problem then becomes that of reconstructing a single, non-periodic object (the contents of the unit cell) from the amplitude of its Fourier transform, which is known to have a unique solution that can be found using iterative phase retrieval algorithms $[3,4]$.

However, if there is more than one molecule per unit cell then the inter-Bragg diffraction from such crystals will depend on the particular configuration of the molecules on the crystal surface, as different unit cells can be defined for different surface terminations. To a first approximation, the diffraction of the unit cell recovered from the method described above is no longer that of a single kind of unit cell but is equal to the incoherent average over a set of unit cells that contain different arrangements of the molecule based on the space group at hand $[5,6]$.

Following from the recent experimental success of shape transform phasing of synthetic crystals without randomly terminated edges [7], the applicability of direct iterative phase retrieval when multiple unit cells are present is explored using experimental data collected from synthetic crystals with random edge terminations at the FERMI free-electron laser in Trieste, Italy. Results so-far indicate successful phase retrieval under this circumstance is still possible.

\section{References}

[1] H.N. Chapman et al., Nature, 470, 73-78 (2011).

[2] J.C.H. Spence et al., Opt. Express, 19, 2866-2873 (2011).

[3] R.P. Millane, J. Opt. Soc. Am. A, 7, 394-411 (1990).

[4] S. Marchesini, Rev. Sci. Instrum., 78, 011301 (2007).

[5] J.P.J. Chen and R.P. Millane, J. Opt. Soc. Am. A, 31, 1730-1737 (2014).

[6] R.A. Kirian et al., Phil. Trans. R. Soc. B, 369, 20130331 (2014).
[7] R.A. Kirian et al., Phys. Rev. X, 5, 011015 (2015).

Keywords: phase retrieval, shape transform, iterative projection algorithms, nanocrystal, edge effects 